\title{
Development of test scenarios and bicyclist surrogate for the evaluation of bicyclist automatic emergency braking systems
}

\author{
Qiang Yi, Stanley Chien, Lingxi Li, Wensen Niu and Yaobin Chen \\ Department of Electrical and Computer Engineering, Indiana University-Purdue University, Indianapolis, Indiana, USA \\ David Good \\ School of Public and Environmental Affairs, Indiana University, Bloomington, Indiana, USA \\ Chi-Chih Chen \\ Department of Electrical and Computer Engineering, Ohio State University, Columbus, Ohio, USA, and \\ Rini Sherony \\ Toyota Collaborative Safety Research Center, Ann Arbor, Michigan, USA
}

\begin{abstract}
Purpose - To support the standardized evaluation of bicyclist automatic emergency braking (AEB) systems, test scenarios, test procedures and test system hardware and software tools have been investigated and developed by the Transportation Active Safety Institute (TASI) at Indiana University-Purdue University Indianapolis. This paper aims to focus on the development of test scenarios and bicyclist surrogate for evaluating vehicle-bicyclist AEB systems.

Design/methodology/approach - The harmonized general estimates system (GES)/FARS 2010-2011 crash data and TASI 110-car naturalistic driving data (NDD) are used to determine the crash geometries and environmental factors of crash scenarios including lighting conditions, vehicle speeds, bicyclist speeds, etc. A surrogate bicyclist including a bicycle rider and a bicycle surrogate is designed to match the visual and radar characteristics of bicyclists in the USA. A bicycle target is designed with both leg pedaling and wheel rotation to produce proper micro-Doppler features and generate realistic motion for camera-based AEB systems.

Findings - Based on the analysis of the harmonized GES/FARS crash data, five crash scenarios are recommended for performance testing of bicyclist AEB systems. Combined with TASI 110-car naturalistic driving data, the crash environmental factors including lighting conditions, obscuring objects, vehicle speed and bicyclist speed are determined. The surrogate bicyclist was designed to represent the visual and radar characteristics of the real bicyclists in the USA. The height of the bicycle rider mannequin is $173 \mathrm{~cm}$, representing the weighted height of 50 th percentile US male and female adults. The size and shape of the surrogate bicycle were determined as 26-inch wheel and mountain/road bicycle frame, respectively. Both leg pedaling motion and wheel rotation are suggested to produce proper micro-Doppler features and support the camera-based AEB systems.

Originality/value - The results have demonstrated that the developed scenarios, test procedures and bicyclist surrogate will provide effective objective methods and necessary hardware and software tools for the evaluation and validation of bicyclist AEB systems. This is crucial for the development of advanced driver assistance systems.
\end{abstract}

Keywords Bicyclist, Surrogate bicyclist, Automatic emergency braking (AEB), Crash scenarios, Crash testing, Naturalistic driving, Radar cross section (RCS), Micro-Doppler

Paper type Research paper

\section{Introduction}

Bicyclist safety has attracted increasing attention by the public, government agencies and transportation and automotive industry as a public safety and health issue. According to the crash data of US Fatality Analysis Reporting System (FARS), 818 bicyclists were killed, and 45,000 bicyclists were injured in

The current issue and full text archive of this journal is available on Emerald Insight at: www.emeraldinsight.com/2399-9802.htm

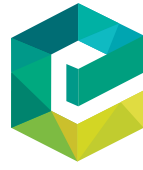

Journal of Intelligent and Connected Vehicles $1 / 1(2018) 15-27$

Emerald Publishing Limited [ISSN 2399-9802] [DOI 10.1108/JICV-02-2018-0005] (c) Qiang Yi, Stanley Chien, Lingxi Li, Wensen Niu, Yaobin Chen, David Good, Chi-Chih Chen and Rini Sherony. Published in fournal of Intelligent and Connected Vehicles. Published by Emerald Publishing Limited. This article is published under the Creative Commons Attribution (CC BY 4.0) licence. Anyone may reproduce, distribute, translate and create derivative works of this article (for both commercial and non-commercial purposes), subject to full attribution to the original publication and authors. The full terms of this licence may be seen at http://creativecommons.org/licences/by/4.0/legalcode

The authors would like to thank Toyota's Collaborative Safety Research Center for the financial and technical support of this research.

Received 27 February 2018

Revised 12 April 2018

23 April 2018

Accepted 24 April 2018 
motor vehicle traffic-related crashes in 2015. The number of bicyclist fatalities in 2015 was about 12.2 per cent higher than that in 2014 (NHTSA, 2018). Figure 1 shows the trends of the bicyclist fatalities and the percentage of bicyclist fatalities in all traffic fatalities since 1994. It is easy to see that the number of bicyclist fatalities has been increasing since 2010. A total number of 840 bicyclists were killed in crashes in 2016 (NHTSA, 2016), which is the highest number of bicyclist fatalities since 1991. This increase might be because of increase in the number of bicyclists between 2012 and 2017, from around 51 million to slightly more than 66 million (Statista, 2018).

Bicyclist protection has become an important issue for traffic safety considerations. In the past, efforts were made to require bicyclists to wear helmets and adhere to riding regulations (MacAlister and Zuby, 2015). Some studies also concluded that some bicyclist safety specific facilities, such as bike routes, bike lanes, bike paths, cycle tracks at roundabouts, could potentially reduce the number of bicycle-related crashes and fatalities. Street lighting, paved surfaces and low-angled grades are additional factors that appear to improve bicyclist safety (Reynolds et al., 2009). While the improvement of the road environment can reduce the risk of bicyclist crashes, the vehicles equipped with crash warning/avoidance systems have also been introduced in recent years to reduce the potential bicyclist injuries and fatalities (Rosen, 2013). These systems are typically referred to as bicyclist pre-collision systems (PCS) or bicyclist automatic emergency braking (AEB) systems. The bicyclist AEB systems are designed to warn the driver and/or brake automatically to help mitigate or avoid imminent bicyclist crashes if the driver does not apply brakes in emergency situations.

To support the development of standardized evaluation of the bicyclist AEB systems, a team of researchers in the Transportation Active Safety Institute (TASI) at Indiana University-Purdue University Indianapolis conducted a twoyear research project in collaboration with Toyota's Collaborative Safety Research Center. One of the main research goals was to develop the test scenarios, test equipment and test procedures for testing and evaluating bicyclist AEB systems (Yi et al., 2016, 2017). During the same period, the European New Car Assessment Programme (Euro NCAP) was also developing a bicyclist AEB testing system, named CyclistAEB Testing System (CATS). Their goal was to draft the test protocols, test scenarios and surrogate target for Cyclist-AEB systems in 2018 and 2020 (den Camp et al., 2017).

Figure 1 Trends of bicyclist fatalities and fatality rates according to FARS

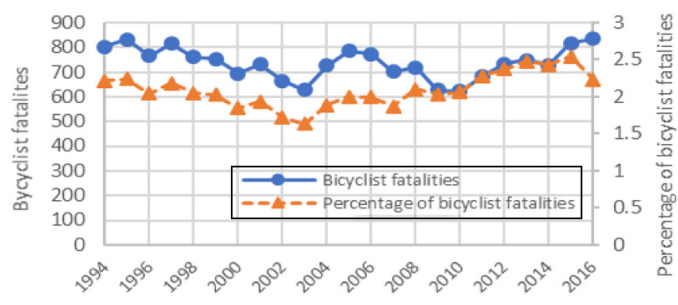

Year
This paper presents the development of test scenarios, surrogate bicyclist and associated hardware and software tools for the evaluation of bicyclist AEB systems. Accidentology is used to determine the primary crash scenarios. The scenario variables are selected based on the national crash databases and the TASI 110-car naturalistic driving data (NDD). To conduct the AEB performance testing, the surrogate bicyclist was designed and developed, which includes a bicycle rider and a bicycle. Its design matches the visual and radar characteristics of the real bicyclists in the USA. The surrogate bicyclist is designed with both pedaling motion and wheel rotation to support the camera and radar detection. The size and clothing color of bicycle riders are determined based on the general estimates system (GES)/FARS crash data and the TASI 110car NDD.

The remainder of this paper is organized as follows. Section 2 introduces the data sources used for generating crash scenarios. Based on the detailed analysis of the crash scenarios, a set of five crash geometries is recommended for the evaluation of bicyclist AEB systems in Section 3. Section 4 discusses environmental factors related to crash scenarios, including lighting conditions, obscuring objects, vehicle speed and bicyclist speed. Section 5 describes the design of the surrogate bicyclist including the determination of the key parameters used in the design of the surrogate bicyclist. Section 6 presents the experimental setup for the proposed bicyclist AEB system test. The conclusion is drawn in Section 7.

\section{Data sources}

Accidentology is used to determine the primary crash scenarios. A crash scenario is defined by three groups of factors:

1 Bicyclist/vehicle crash geometries;

2 Bicyclist crash environmental factors including lighting conditions, obscuring objects, vehicle speed and bicyclist speed; and

3 Bicyclist description factors including size, bicyclist clothing color and bicyclist limb motion.

The test scenario analysis was based on three data sources, the GES, FARS and TASI 110-car NDD. The GES data include a nationally representative sample data set of approximately 50,000 police-reported motor vehicle related bicyclist crashes of all severities. FARS is a census of all fatal motor vehicle crashes that occurred in the USA.

First, all bicycle-related records in the harmonized GES/FARS 2010-2011 were extracted, which included approximately 55,000 crashes, 693 fatalities and US $\$ 10.08$ bn in social cost. Considering the fact that many crashes were not AEB relevant, the data set was reconstructed to exclude the following conditions:

- crashes involving more than one bicyclist;

- crashes involving many vehicles, heavy vehicles or motorcycles; and

- first harmful event is not the collision with a bicyclist; and

- the driver backed over or lost control.

After removing the irrelevant cases, or cases that are too difficult to simulate, approximately 38,816 crashes per year (about 71.5 per cent of all bicyclist crashes and approximately 
70.5 per cent of all bicyclists involved), 481 fatalities per year (approximately 69.4 per cent of all bicyclist fatalities) and US $\$ 7.06$ bn in social cost per year (70.0 per cent of bicyclist injury-related social costs) were retained as AEB-relevant and testable.

Although the GES/FARS data could provide a reasonable estimate of crash geometries, some important details required for creating test scenarios were missing, such as the bicycle moving speed, the bicyclist clothing color and the bicycle limb motion pattern. To determine these parameters, TASI 110-car NDD were used.

The TASI 110-car NDD were collected and processed. The database has naturalistic driving information of 116 drivers that was recorded from February 2012 to June 2013. Each driver completed a 12-month NDD collection process. About 90 terabytes of data were collected with near $40,000 \mathrm{~h}$ and 1.44 million miles of driving information. The NDD provided the front view in $1080 \mathrm{p}$ resolution video, GPS locations, 3D acceleration and the vehicle speed of each vehicle in a one-year period. Hence, the TASI 110-car NDD helped us identify the missing parameters from the GES/ FARS data.

\section{Crash geometries}

The most important characteristic of the vehicle-bicycle crashes is the relative trajectories of the vehicle and bicycle. Based on the harmonized GES/FARS 2010-2111 crash data, the basic crash geometries consist of 13 bicycle crossing road scenarios and 11 bicycle parallel to the car scenarios, as shown in Figure 2. The coding scheme in Figure 2 is based on three key features of the crash geometry. The first component includes the paths of the vehicle and the bicycle before the crash. Where "PP" means bicycle path is parallel to vehicle path, and "CP" stands for bicycle crossing road and vehicle along the road paths. "UP" means unknown. "XX" is for crashes that are not bicyclist AEB system-relevant, such as crashes involving a parked vehicle. The second component is vehicle action, where "VS", "VRT", "VLT", "VHO", "VOT", "VDO" and "VDT" represent the vehicle going straight, the vehicle right turning, the vehicle left turning, the vehicle head-on approaching the bicycle, the vehicle overtaking the bicycle, the vehicle driving out (stop and drive out without yielding) and the vehicle driving through (ignoring potential stops), respectively. The third component is the bicyclist behavior, where "BS", "BOT", "BHO", "BRT" and "BLT" represent the bicyclist going straight, the bicyclist overtaking the vehicle, the bicyclist striking the vehicle head-on, the bicyclist right turning and the bicyclist left turning, respectively. All these abbreviations and their definitions can be found in Table I.

These harmonized data with bicycle crash types provide an opportunity to obtain the most detailed crash information about bicyclists in both absolute and relative crash geometries. To determine the detailed testing scenarios, vehicle pre-crash maneuver and bicyclist behavior (Figure 2) are discussed as follows.

\subsection{PP:VOT, parallel crashes with the vehicle traveling straight and overtaking the bicycle}

The scenarios with the bicycle being overtaken by vehicle represent the crashes that do not happen at intersections. Consequently, the vehicle speed could be represented by a function related to the speed limit of the road. The term BCType of GES/FARS provides details for decomposing the PP:VOT into subtypes by incorporating bicycle behavior beyond the direction of travel (Figure 3). The subtypes include the bicycle turning right in front of the vehicle (PP:VOT-BRT), the bicycle turning left in front of the

Figure 2 Basic crash geometries

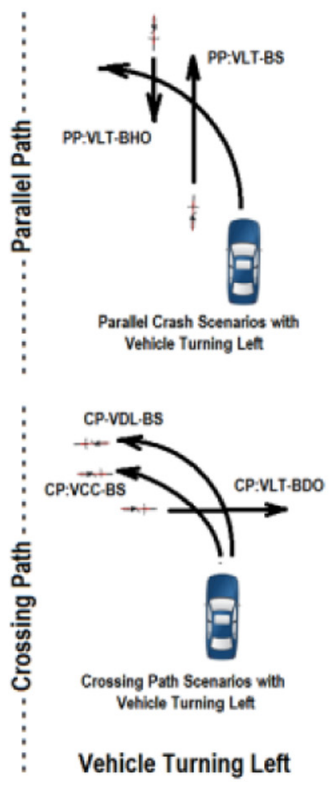

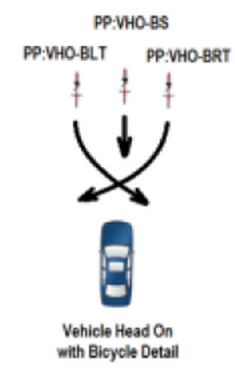
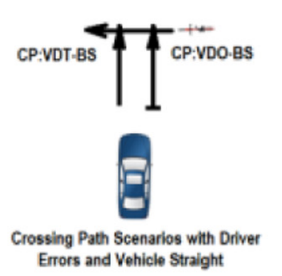

Vehicle Travelin

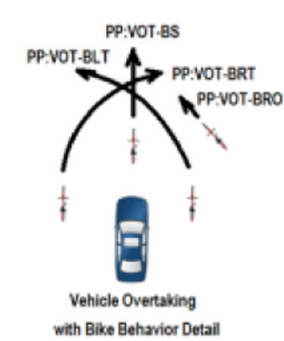

with Bicycle Detail

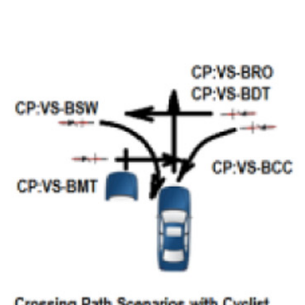

Crossing Path Scenarios with Cyclist
Enrons and Vehicle Straight

Emons and Vehicle Straight

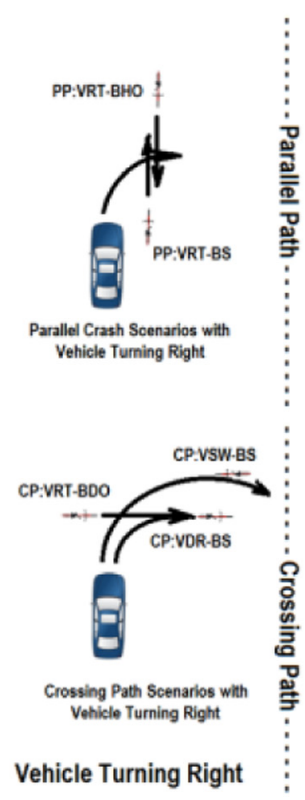


Table I Abbreviations/definitions

\begin{tabular}{ll}
\hline Abbreviation & Definition \\
\hline PP & Bicycle path is parallel to vehicle path \\
CP & Bicycle crossing road and vehicle along the road path \\
UP & Unknown paths \\
XX & Crashes that are not bicyclist AEB system relevant, \\
& such as crashes involving a parked vehicle \\
VS & Vehicle going straight \\
VRT & Vehicle right turning \\
VLT & Vehicle left turning \\
VHO & Vehicle head-on approaching the bicycle \\
VOT & Vehicle overtaking the bicycle \\
VDO & Vehicle driving out (stop and drive out without yielding) \\
VDT & Vehicle driving through (ignoring potential stops) \\
BS & Bicyclist going straight \\
BOT & Bicyclist overtaking the vehicle \\
BHO & Bicyclist striking the vehicle head-on \\
BRT & Bicyclist right turning \\
BLT & Bicyclist left turning \\
\hline
\end{tabular}

Figure 3 Crash geometry of PP:VOT scenarios

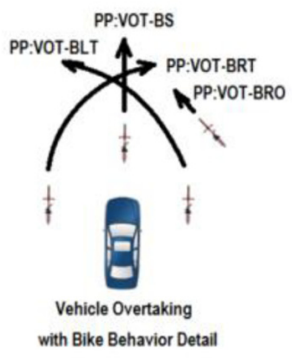

vehicle (PP:VOT-BLT), the bicycle traveling straight and struck from behind by the vehicle (PP:VOT-BS) and the bicycle riding out from a midblock location (PP:VOTBRO).

Table II shows the data of crash geometries PP:VOT. The overall social cost in this group is US $\$ 1812.22 \mathrm{~m}$, covering about 26 per cent of the total annual social cost. In the subscenarios, PP:VOT-BS comprises the maximum percentage of the vehicle overtaking crashes; it covers approximately 19 per cent of the total social cost of all bike crashes. It should be noticed that "Fatalities" in Table II is annualized fatalities, and the "per cent" is the percentage of social cost in all crash cases. The unit for social cost is million US dollars.

Table II Crashes, fatalities and social cost of PP:VOT

\begin{tabular}{lcccr}
\hline Scenarios & Crashes & Fatalities & Social cost & $(\%)$ \\
\hline PP:VOT-BLT & 960 & 29.5 & 360.83 & 5.2 \\
PP:VOT-BRO & 441 & 4.5 & 77.59 & 1.1 \\
PP:VOT-BRT & 342 & 6.5 & 67.95 & 1.0 \\
PP:VOT-BS & 2,609 & 118.5 & $1,311.85$ & 18.7 \\
Overall PP:VOT & 4,352 & 159.0 & $1,812.22$ & 26.0 \\
\hline
\end{tabular}

\subsection{PP:VHO, vehicle going straight and has head-on collisions with the bicycle}

Head-on collisions are typically not associated with intersections and both bicyclist and vehicle travel at reasonably high speeds (Figure 4). Details for head-on crashes include:

- Bicyclist is making a right turn (PP:VHO-BRT), where it is initially on the opposite side of the road.

- Bicyclist is initially traveling on the same side of the road as the vehicle but does not leave sufficient gap for the vehicle (PP:VHO-BLT).

- Vehicle is traveling on the wrong side of the road, or the bicycle is traveling on the wrong side of the road (PP:VHO-BS).

Since both mistakes involve the same crash geometries, they are combined into one single scenario. The results are shown in Table III. It covers about 4.3 per cent of the annual social cost.

\subsection{CP:VDO, crossing paths with vehicle going straight} without the right of way

This type of crashes represents scenarios that the vehicle is traveling straight and does not have the right of way (Figure 5). The detailed sub-scenario types are shown in Table IV. This group represents about 13 per cent of the bicycle crashes and less than 1 per cent of the fatalities. Two different sub-scenario types are found: 1) CP:VDO-BS (driver stops and goes and fails to yield) and CP:VDT-BS (driver does not stop), which represent 3.9 and 1.2 per cent of the total social cost, respectively.

3.4 CP:VS, crossing paths with vehicle traveling straight with right of the way

This type of crashes represents the scenarios where the vehicle is traveling straight and has the right of way

Figure 4 Crash geometry of PP:VHO scenarios

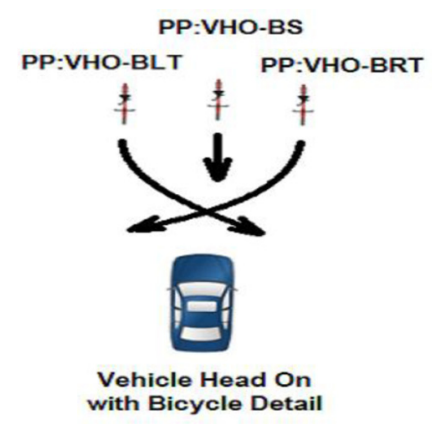

Table III Crashes, fatalities and social cost of PP:VHO

\begin{tabular}{lcccc}
\hline Scenarios & Crashes & Fatalities & Social cost & $(\%)$ \\
\hline PP:VHO-BHO & 510 & 20.5 & 221.43 & 3.2 \\
PP:VHO-BLT & 306 & 5.0 & 66.65 & 1.0 \\
PP:VHO-BRT & 37 & 1.0 & 14.96 & 0.0 \\
Overall PP:VHO & 853 & 26.5 & 303.04 & 4.3 \\
\hline
\end{tabular}


Figure 5 Crash geometry of CP:VDO scenarios

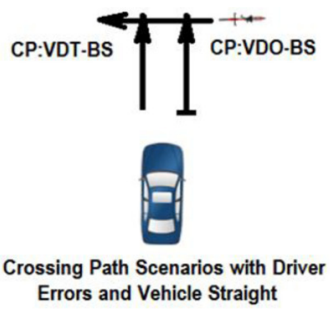

Table IV Crashes, fatalities and social cost of CP:VDO

\begin{tabular}{lcccc}
\hline Scenarios & Crashes & Fatalities & Social cost & $(\%)$ \\
\hline CP:VDO-BS & 4,183 & 3.5 & 273.05 & 3.9 \\
CP:VDT-BS & 907 & 3.5 & 84.45 & 1.2 \\
Overall CP:VDO & 5,090 & 7.0 & 357.51 & 5.1 \\
\hline
\end{tabular}

(Figure 6). It is expected that the vehicle travels within the speed limit. This type of crashes is very complicated. Except for the sub-scenarios of bicycle riding through (CP:VS$\mathrm{BDT})$ and riding out (CP:VS-BRO), the crashes involve the bicyclist cutting the corner (CP:VS-BCC), the bicyclist swinging wide (CP:VS-BSW), the bicyclist is trapped or there is an obscuring object (CP:VS-BMT) and other cases where the bicyclist has turning errors (CP:VS-BTE). The results are shown in Table $\mathrm{V}$. We can find that the scenario of CP:VS-BRO has the highest percentage in terms of the social cost, which covers about 12.5 per cent of the total social cost.

Figure 6 Crash geometry of CP:VS scenarios

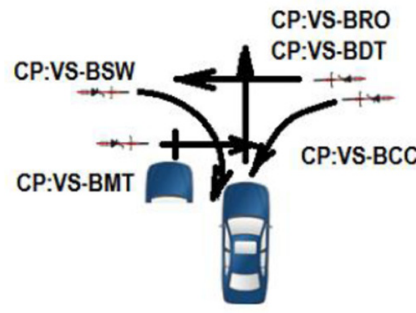

Crossing Path Scenarios with Cyclist Errors and Vehicle Straight

Table V Crashes, fatalities and social cost of CP:VS

\begin{tabular}{lcccr}
\hline Scenarios & Crashes & Fatalities & Social cost & $(\%)$ \\
\hline CP:VS-BRO & 3,628 & 60 & 877.58 & 12.5 \\
CP:VS-BDT & 2,236 & 52 & 637.88 & 9.1 \\
CP:VS-BMT & 193 & 2.5 & 30.22 & 0.0 \\
CP:VS-BCC & 179 & 2 & 30.16 & 0.0 \\
CP:VS-BSW & 122 & 1 & 16.37 & 0.0 \\
CP:VS-BTE & 12 & 0.5 & 5.25 & 0.0 \\
Overall CP:VS & 6,358 & 116.5 & $1,592.20$ & 22.7 \\
\hline
\end{tabular}

\subsection{PP:VLT, parallel scenarios with the vehicle turning} left

This type of crashes is shown in Figure 7, which has two common sub-scenario types:

1 vehicle turning left and the bicyclist traveling in the same direction as the vehicle before it turns (PP:VLT-BS); and

2 bicyclist on the road or on the sidewalk enters the intersection, while the vehicle turns left into the roadway or a junction (PP:VLT-BHO).

These two types of crashes comprise about 8 per cent of all bicyclist crashes and 4.8 per cent of the total social cost (Table VI).

3.6 CP:VLT, crossing scenarios with vehicle turning left The second type of vehicle left-turn scenarios is associated with bicycle crossing paths (Figure 8). This type of crashes includes:

- vehicle does not yield to a bicycle (CP:VDL-BS);

- driver cuts the corner and strikes a bicyclist (CP:VCC-BS);

- bicycle drives out (CP:VLT-BDO); and

- other unidentified cases.

Figure 7 Crash geometry of PP:VLT scenarios

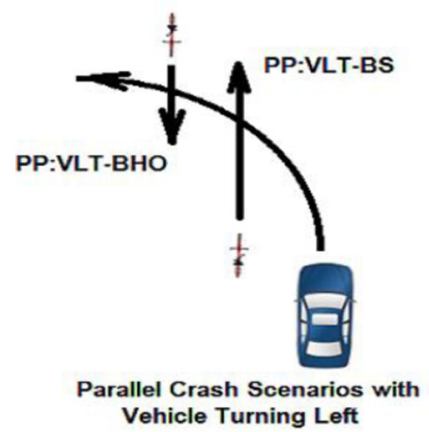

Table VI Crashes, fatalities and social cost of PP:VLT

\begin{tabular}{lcccc}
\hline Scenarios & Crashes & Fatalities & Social cost & $\mathbf{( \% )}$ \\
\hline PP:VLT-BHO & 3,076 & 9.0 & 334.51 & 4.8 \\
PP:VLT-BS & 597 & 3.0 & 76.18 & 1.1 \\
Overall PP:VLT & 3,674 & 12.0 & 410.69 & 5.9 \\
\hline
\end{tabular}

Figure 8 Crash geometry of CP:VLT scenarios

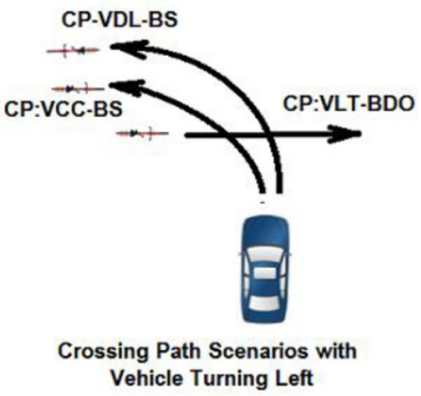


As this type of crashes only covers about 1.9 per cent of the total social cost, they are not considered in the AEB system evaluation (Table VII).

\subsection{CP:VRT, crossing scenarios with vehicle turning right}

This type of crashes occurs when the vehicle turns right and bicyclist crosses (Figure 9). It covers about 8 per cent of the total social cost. The most frequent scenario in this type is the situation where the vehicle swings too wide as they make the right turn (CP:VSW-BS). It covers 3.1 per cent of the total social cost. The second most frequent crash scenario is CP: VDR-BS, where the vehicle "drives out" while making a right turn and bicyclist goes straight and crosses the road (Table VIII).

\subsection{PP:VRT, parallel scenarios with the vehicle turning right}

This type of crashes involves parallel path crashes with the vehicle turning right. They consist of two bicyclist crash scenarios: PP:VRT-BS and PP:VRT-BHO. As both of them have a small percentage of the total social cost (2.2 and 1.0 per cent, respectively), they are not considered in the AEB system evaluation (Figure 10) (Table IX).

Table VII Crashes, fatalities and social cost of CP:VLT

\begin{tabular}{lcccc}
\hline Scenarios & Crashes & Fatalities & Social cost & $(\%)$ \\
\hline CP:VCC-BS & 1,273 & 3.0 & 95.81 & 1.4 \\
CP:VLT-BDO & 267 & 0.5 & 21.80 & 0.0 \\
CP:VDL-BS & 287 & 0.0 & 14.51 & 0.0 \\
CP:VLT-Otr & 557 & 0.0 & 31.77 & 0.5 \\
Overall CP:VLT & 2,384 & 3.5 & 163.89 & 1.9 \\
\hline
\end{tabular}

Figure 9 Crash geometry of CP:VRT scenarios

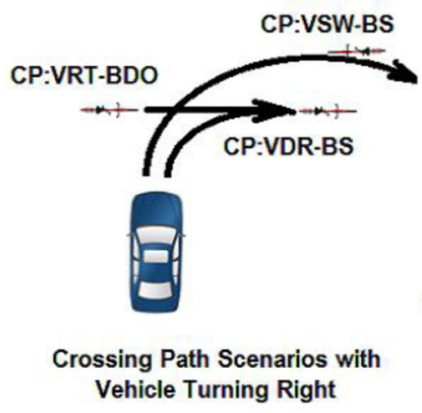

Table VIII Crashes, fatalities and social cost of CP:VRT

\begin{tabular}{lcccc}
\hline Scenarios & Crashes & Fatalities & Social cost & (\%) \\
\hline CP:VDR-BS & 3,209 & 2.0 & 214.08 & 3.1 \\
CP:VSW-BS & 3,536 & 3.5 & 239.31 & 3.4 \\
CP:VRT-BDO & 714 & 1.0 & 53.74 & 0.8 \\
CP:VRT-Otr & 688 & 1.5 & 54.93 & 0.8 \\
Overall CP:VRT & 8,147 & 8.0 & 562.06 & 8.0 \\
\hline
\end{tabular}

Figure 10 Crash geometry of PP:VRT scenarios

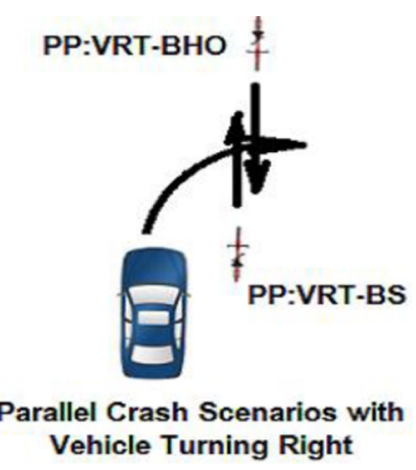

Table IX Crashes, fatalities and social cost of PP:VRT

\begin{tabular}{lcccc}
\hline Scenarios & Crashes & Fatalities & Social cost & $(\%)$ \\
\hline PP:VRT-BS & 1,735 & 2.0 & 153.85 & 2.2 \\
PP:VRT-BHO & 874 & 2.0 & 71.29 & 1.0 \\
Overall PP:VRT & 2,609 & 4.0 & 225.14 & 3.2 \\
\hline
\end{tabular}

\subsection{Recommended crash geometries}

Based on the detailed analysis of the above scenarios, a set of five crash scenarios is recommended for the evaluation of the bicyclist AEB systems. It involves three parallel crash geometries:

1 parallel paths with the vehicle overtaking the bicycle (PP:VOT);

2 parallel paths with the vehicle approaching head-on (PP:VHO); and

3 parallel paths with vehicle turning left and bicycle striking vehicle head-on (PP:VLT-BHO).

It also involves two crossing crash geometries:

1 crossing paths with vehicle driving out (CP:VDO-BS); and

2 crossing paths with vehicle going straight and the bicyclist is riding out (CP:VS-BRO).

While the CP:VS-BDT scenario incorporates a large fraction of overall social cost, it is very similar to the CP:VS-BDT scenario; the only difference being whether the bicycle stops or not prior to its failure to yield at the intersection. This allows the inclusion of the CP:VDT-BS scenario where the vehicle is primarily at fault by failing to yield.

\section{Crash environmental factors}

Besides crash geometries, we need to determine the crash environmental factors, which include lighting conditions, obscuring objects, vehicle speed and bicyclist speed.

\subsection{Lighting conditions}

Three lighting levels are considered: daylight, dark-lit (which includes dusk and dawn) and dark-unlit. The statistical data show that a vast majority of bicyclist crashes occur during the daytime, which cover 61.7 per cent of the total social cost (Table X). 
Qiang Yi et al.

Table X Distributions of different lighting conditions

\begin{tabular}{lrcrr}
\hline Lighting & Crashes & Fatalities & Social cost & (\%) \\
\hline Daylight & 32,945 & 235.0 & $4,318.89$ & 61.7 \\
Dark-lit & 7,397 & 120.0 & $1,643.39$ & 23.5 \\
Dark-unlit & 1,402 & 96.5 & $1,001.65$ & 14.3 \\
Other & 72 & 3.0 & 39.03 & 0.6 \\
\hline
\end{tabular}

\subsection{Obscuring objects}

Our analysis of obscuring objects considers three major types:

1 physical objects between the driver and the bicyclist, such as a parked car or truck;

2 moving vehicles during a multiple threat/trapped event, which mostly happen in crossing pathway geometries; and 3 glare.

The crashes involving obscuring objects account for less than 10 per cent of all crashes (Table XI). The detailed scenarios suggest that obscuring objects do not play an important role except for the scenario of CP:VS-BMT.

\subsection{Vehicle speed}

There is no data source available to describe vehicle speed for bicyclist-related crashes. We estimate the vehicle crash speed by using the speed limit of the road. The distribution of the speed limit for crashes is shown in Figure 11, where we find that the most important speed limit for crashes is $25 \mathrm{mph}$, while the most important speed limits for fatalities are $35 \mathrm{mph}$ and $45 \mathrm{mph}$.

\subsection{Bicyclist speed}

From the crash databases, it is difficult to estimate bicycle speed during crashes. TASI 110-car NDD were used for finding bicyclist speed (Fu et al., 2017). From the data, 1,000

Table XI Distributions of different obscuring objects

\begin{tabular}{lrccr}
\hline Obstructions & Crashes & Fatalities & Social cost & $(\%)$ \\
\hline None & 37,694 & 421.5 & $6,406.53$ & 91.5 \\
Object & 2,593 & 12.5 & 292.34 & 4.2 \\
Glare & 1,195 & 11.5 & $1,89.63$ & 2.7 \\
Other & 334 & 9.0 & $1,14.47$ & 1.6 \\
\hline
\end{tabular}

Figure 11 Distribution of speed limits for crashes

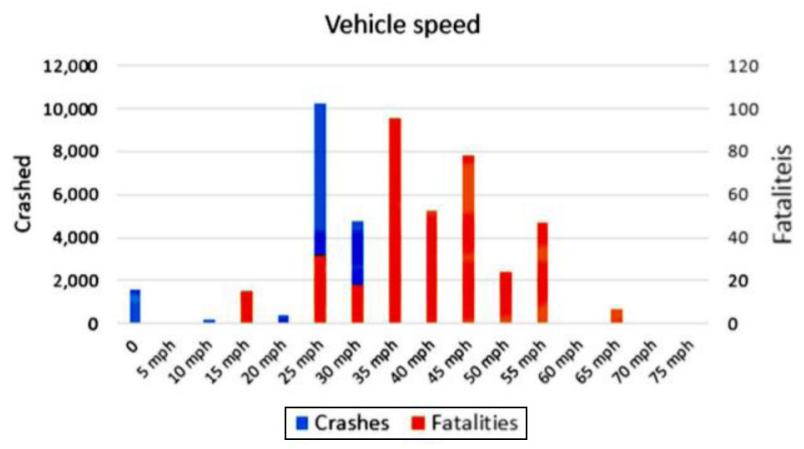

bicyclist cases are obtained through video analysis. Three main scenarios are analyzed:

1 bicyclist moving along the road;

2 bicyclist crossing the road with constant speed (ride through); and

3 bicyclist crossing the road from stationary (ride out).

The bicycle moving speed distributions for the scenarios of moving along the road and ride through crossing the road are shown in Figures 12 and 13, respectively. For the scenario of moving along the road, the average bicyclist moving speed is $5.59 \mathrm{~m} / \mathrm{s}$. The 25 th and 75 th percentile speeds are $4.06 \mathrm{~m} / \mathrm{s}$ and $6.94 \mathrm{~m} / \mathrm{s}$, respectively. For the scenario of ride through crossing the road, the average moving speed is $5.23 \mathrm{~m} / \mathrm{s}$, and the 25 th and 75th percentile speeds are $3.94 \mathrm{~m} / \mathrm{s}$ and $6.26 \mathrm{~m} / \mathrm{s}$, respectively. The scenario of crossing the road from being stationary is more complex. The overall average moving speed is $3.5 \mathrm{~m} / \mathrm{s}$. To obtain detailed moving behavior, the road is marked with five key points: roadside, 25 per cent of the road, 50 per cent of the road, 75 per cent of the road and another side of the road. The average speeds when crossing the road from stationary at 25,50 and 75 per cent of the road are $2.95 \mathrm{~m} / \mathrm{s}$, $3.77 \mathrm{~m} / \mathrm{s}$ and $3.85 \mathrm{~m} / \mathrm{s}$, respectively. The average bicyclist crossing the road acceleration is $1.4 \mathrm{~m} / \mathrm{s}^{2}$.

Figure 12 Speed distributions for the bicyclist moving along the road

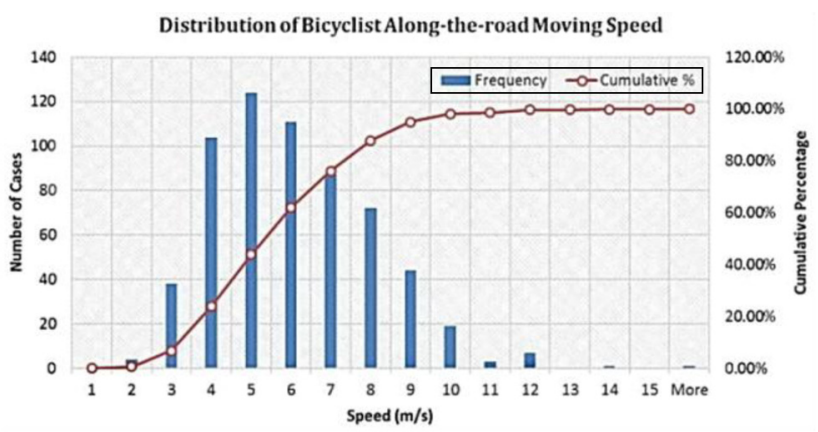

Source: Fu et al. (2017)

Figure 13 Speed distributions for the bicyclist ride through crossing the road

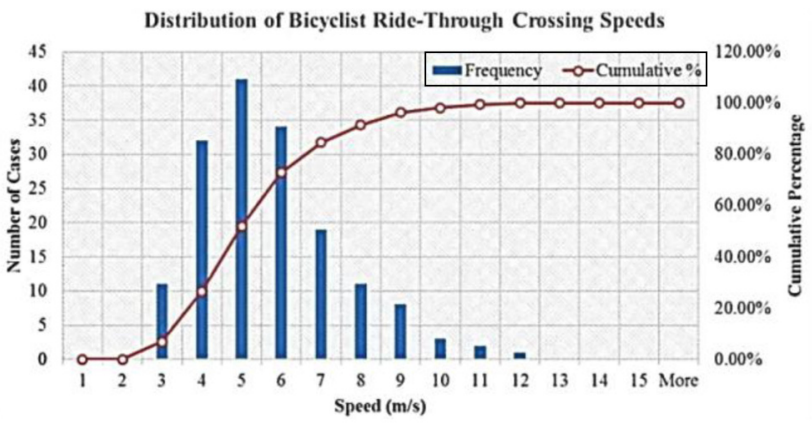

Source: Fu et al. (2017) 


\section{Surrogate bicyclist design}

Bicyclist crash geometries, bicyclist crash environmental factors and surrogate bicyclist design are the three key variables that affect the performance testing of AEB systems. This section presents the detailed design of the surrogate bicyclist system.

\subsection{Representative size of bicycle riders and bicycles}

According to "Bicycling and Walking in the USA, 2014 Benchmarking Report", adults between the age of 16 and 64 account for 77 and 68 per cent of all bicyclist fatalities and injuries, respectively (Milne and Melin, 2014). Seniors of age 65 or higher represent 12 per cent of all bicyclist fatalities and 5 per cent of all bicyclist injuries. Children under age of 16 represent 11 per cent of all bicyclist fatalities and 27 per cent of all bicyclist injuries between 2009 and 2011. As bicyclists over the age of 16 cover 89 per cent of fatalities and 73 per cent of injuries, only the adult surrogate bicyclist size is recommended. Moreover, only the male adult-sized bicyclist surrogate is recommended, as male account for 87 per cent of bicyclist fatalities and 83 per cent of bicyclist injuries.

For scientific soundness, a more precise method to determine the size of the surrogate is the weighted 50th percentile size of male and female adults. Therefore, the weighted height of US male and female adults, $173 \mathrm{~cm}$, is recommended as the height of bicycle rider surrogate. As a few centimeters height difference might not affect the bicyclist detection significantly, traditional 50th percentile height of US male adults, $175.6 \mathrm{~cm}$ (CDC, 2018), is also acceptable as the height of surrogate bicycle rider.

According to the statistical report of Bicycle Product Supplier Association in 2012, the most popular adult bicycles in US market have 26-inch wheel size. The frame types of most adult bicycles are mountain bicycles and road bicycles. Therefore, the wheel size of the adult surrogate bicycle is suggested to be 26 inches, and the shape of the adult surrogate bicycle is defined as a mix of mountain and road types.

\subsection{Limb motion of the bicycle rider}

Limb motion of bicyclists can be a useful feature for bicyclist detection. Many camera-based pedestrian and bicyclist detection studies emphasize the importance of limb motion for achieving better detection results (Wojek et al., 2009; Takahashi et al., 2010). For the radar-based detection systems, it was also pointed out that the limb motion/pedaling can produce a very noticeable micro-Doppler effect from the front $\left(0^{\circ}\right)$, back $\left(180^{\circ}\right)$ and $45^{\circ}$-side observation angles, as shown in Figure 14 (Belgiovane and Chen, 2017).

There has been some discussion about whether the limb motion/pedaling is required for the surrogate bicyclist. Euro NCAP has adopted the bicyclist surrogate with fixed leg posture (both legs bent) based on their study that suggests that a majority of bicyclists (over 80 per cent) stop pedaling when crossing an intersection in Europe. By examining 484 randomly selected bicyclist video clips in the TASI 110-car NDD (Sherony et al., 2016), it is observed that 83.2 per cent of bicyclists have pedaling motion when crossing the road, and 100 per cent of bicyclists have pedaling motion when moving along the road. The average pedaling frequency is $0.85 \mathrm{~Hz}$ for the cases of crossing the road (CP:VDO and CP:VS-BRO) and
Figure 14 Micro-Doppler effect of pedaling motion

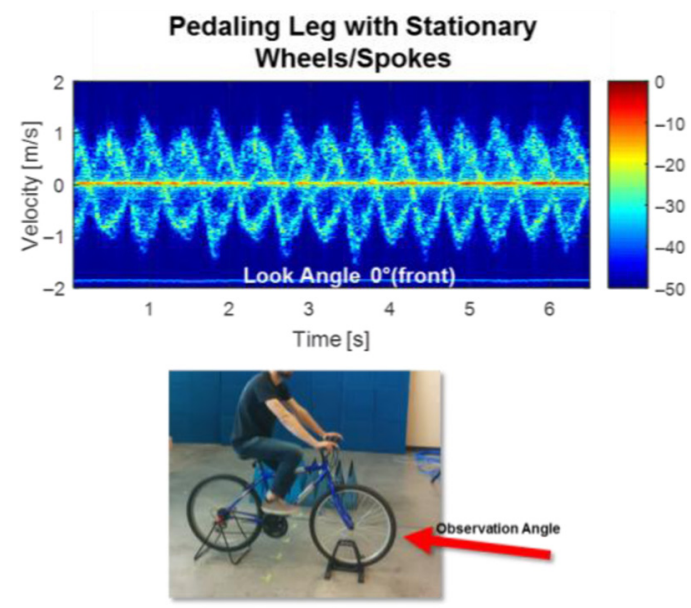

$1.01 \mathrm{~Hz}$ for the cases of moving along the road (PP-VOT and PP-VHO).

To demonstrate the importance of pedaling motion in the performance of bicyclist AEB system, a comparison AEB test of the same surrogate bicyclist with two different leg postures (one straight leg or both bending legs) was conducted [Figure 15(a) and Figure 15(b)]. The test vehicle used was a commercially available SUV that has a radar and camera-based bicyclist AEB system. The results [plotted in Figure 15(c)] show that the AEB system performs better for the cases where one leg is straight than for cases where both legs are bent. The results demonstrate that leg postures do affect the performance of bicyclist AEB systems. The same conclusion was also reported

Figure 15 Comparison of bicyclist AEB system performance with different leg postures

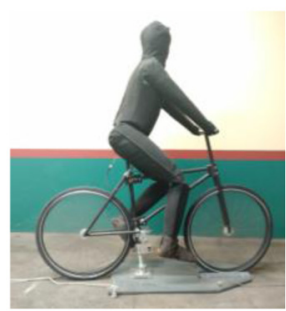

(a)

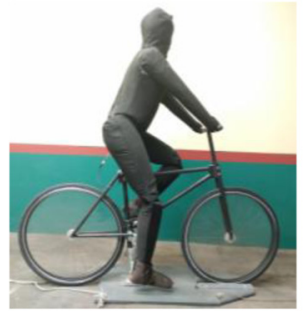

(b)

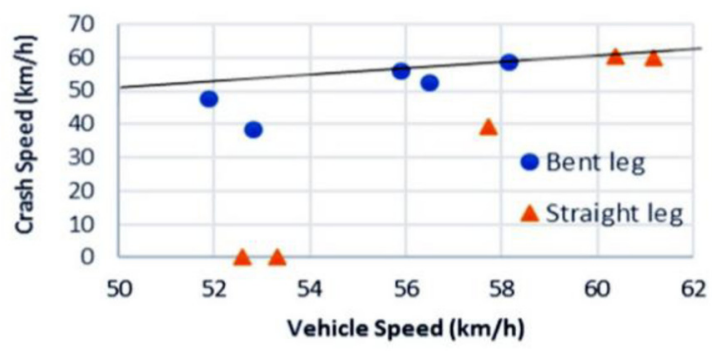

(c)

Notes: (a) Leg posture 1 (bent); (b) leg posture 2 (straight); (c) vehicle speed vs crash speed 
by a study from Euro NCAP (den Camp et al., 2017). The possible reason of performance difference given in den Camp et al. (2017) is that the AEB system uses characteristics from the $360^{\circ}$ pedaling sequence. Based on the above discussions, the leg motion/pedaling capability is recommended for the proposed surrogate bicyclist design.

\subsection{Wheel rotation}

Although it is difficult to know if any camera-based AEB system on a production vehicle uses the wheel rotation to detect the bicyclist, it is well documented that radar systems use microDoppler to detect wheel rotation of bicyclists. The pedaling motion and the wheel rotation can generate clear microDoppler responses. The experimental results presented in Belgiovane and Chen (2017) have shown that a rotating wheel could produce distinctive periodic micro-Doppler spectral lines whose fundamental frequency is related to the vehicle speed, and the periodic frequency changes are related to the leg and wheel rotations. This micro-Doppler effect of a wheel can be observed in front $\left(0^{\circ}\right)$, back $\left(180^{\circ}\right)$ and $45^{\circ}$ side view angles. Figure 16 shows the micro-Doppler measurement of rotating bicycle wheel of $66 \mathrm{rpm}$ in the back view. The discrete ripples in the vertical direction reflect the tire thread pattern.

When both wheels can be detected using micro-Doppler, an $\mathrm{H}$-shape micro-Doppler signature can be observed (CDN, 2018; TNO, 2018). This H-shaped bicycle signature cannot be observed when the bicycle is ahead of the vehicle and moving along the road.

As the automotive radar systems can use micro-Doppler characteristics for bicycle detection, the wheel rotation is also recommended for the proposed surrogate bicyclist design.

\subsection{Clothing color of bicycle rider}

The camera is a common sensor used for bicyclist detection. Besides the shape of the bicyclist, the clothing color also significantly affects the performance of bicyclist AEB systems. To ensure that the clothing color identification is not affected by lighting conditions, the images of 1,905 adult bicyclists not under shade were filtered from all bicyclists detected in the TASI 110 car naturalistic driving study. For these bicyclists' images, the K-means clustering algorithm is applied to find the color clusters for both upper and lower cloth colors in the International Commission on Illumination (CIE) LUV color space. As a result, the black/deep gray combination is suggested to be the most representative clothing color for adult bicyclists.

Figure 16 Micro-Doppler measurement of bicycle wheel in $180^{\circ}$ observation angle under the rotation speed of $66 \mathrm{rpm}$
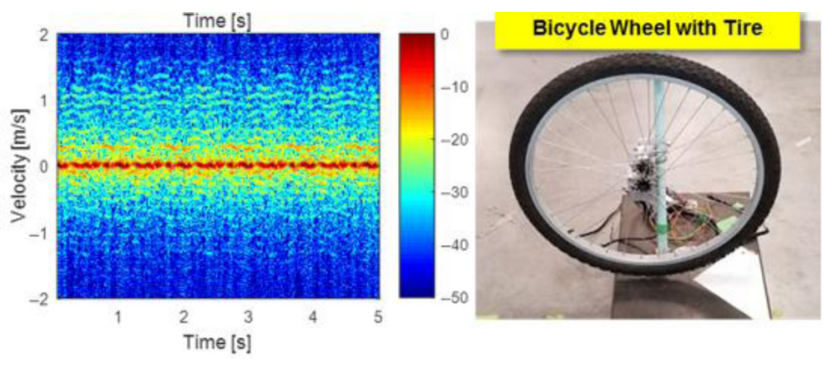

Details of color selection method can be found in den Camp et al. (2017). For the convenience of selecting the color needed, a range of \pm 10 per cent variation of brightness is used to make the acceptable color range. The RGB values and acceptable color ranges for both upper and lower clothing color are shown in Figure 17.

\subsection{Surrogate bicyclist hardware development}

The surrogate bicyclist (including a bicycle rider and a bicycle surrogate) is designed to represent the visual and radar characteristics of the real bicyclists in the USA. Therefore, the surrogate bicyclist should have similar physical properties with respect to most sensors used for the bicyclist detection. The parameters identified and recommended in Sections 5.1-5.4 are considered as part of the requirements for the proposed surrogate bicyclist design.

In 2015, the first generation of the surrogate bicyclist was developed by TASI (Yi et al., 2016). The surrogate bicycle rider has skin that matches the $77-$ to $78-\mathrm{GHz}$ radar reflectivity of the human skin. By using this skin and realistic body shape, the radar cross section (RCS) of the surrogate bicycle rider is similar to representative real bicycle rider from all 360-degree angles in the view of the $77 \mathrm{GHz}$ automotive radar. To harmonize our surrogate bicyclist design with CATS' design, we conducted a comparison study by cross-testing and examining each other's surrogate bicyclist prototype in 2016 . Based on the results, we modified our design and developed a new generation of the harmonized surrogate bicyclist, which consists of three key components: bicycle rider, bicycle and transport sled (Figure 18). Owing to the fact that most adult

Figure 17 Suggested clothing colors for bicyclist AEB systems testing

\begin{tabular}{|c|c|c|c|c|c|}
\hline & Color & $\begin{array}{c}\text { RGB } \\
\text { values }\end{array}$ & $\begin{array}{c}\text { Bright- } \\
\text { ness } \\
\text { variation }\end{array}$ & $\begin{array}{c}\text { Darker } \\
\text { color }\end{array}$ & $\begin{array}{c}\text { Lighter } \\
\text { color }\end{array}$ \\
\hline $\begin{array}{c}\text { Upper } \\
\text { clothing } \\
\text { color }\end{array}$ & $\begin{array}{c}\text { Black } \\
\text { (Deep } \\
\text { grey) }\end{array}$ & $57,60,67$ & $10 \%$ & $49,52,59$ & $65,68,75$ \\
\cline { 6 - 6 } \cline { 5 - 6 } $\begin{array}{c}\text { Lower } \\
\text { clothing } \\
\text { color }\end{array}$ & Black & $44,42,46$ & $10 \%$ & $37,36,39$ & $51,48,53$ \\
\cline { 5 - 6 } & & & & \\
\hline
\end{tabular}

Source: Yi et al. (2017)

Figure 18 Prototype of the surrogate bicyclist

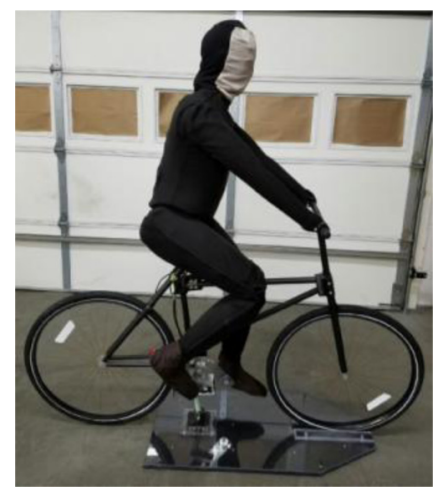


bicyclists involved in crashes in the USA are male, the 50th percentile height of US male population, $175.6 \mathrm{~cm}$, is considered as the surrogate bike rider's height. The mannequin for pedestrian PCS evaluation (Yi et al., 2014) is modified as the bicycle rider. The body and limbs are made to generate realistic human body shape. The harmonized surrogate bicycle has both leg pedaling and wheel rotation to produce the proper micro-Doppler features (Belgiovane and Chen, 2017) and support the camera-based AEB systems (Takahashi et al., 2010)

The finished prototype of the harmonized surrogate bicyclist is shown in Figure 18. The total weight of the surrogate bicycle is $18.4 \mathrm{lbs}(8.4 \mathrm{~kg})$, and the total weight of the surrogate bicycle rider is $9 \mathrm{lbs}(4.1 \mathrm{~kg})$. The crash testing experiments show that a developed target can handle 45-mph full-speed crash without damage or with minor damage and can be reset in $5 \mathrm{~min}$.

\subsection{Radar Cross Section and micro-Doppler effect}

The far-field RCS pattern data of the harmonized surrogate bicyclist are measured. Figure 19(a) shows the comparison between the smoothed ( $7^{\circ}$ average moving) RCS patterns of the US 26-inch mountain bike with a human rider and the prototype harmonized surrogate bicycle and rider mannequin. Figure 19(b) compares the measured RCS patterns of the 26-inch mountain bike and the harmonized surrogate bicycle. It can be seen that the measured RCS data of the developed surrogate bicyclist agree well with that of the real rider plus bicycle in terms of both RCS pattern shape and level in 360 degrees.

Figures 20 and 21 show the micro-Doppler measurement of leg pedaling and wheel rotation produced by the developed surrogate bicyclist. The micro-Doppler features match the real human leg pedaling shown in Figure 14, and the real wheel rotation is shown in Figure 16.

Figure 19 Smoothed ( $7^{\circ}$ moving average) RCS patterns for real bicycle + human and mannequin + surrogate bike under the $77 \mathrm{GHz}$ radar
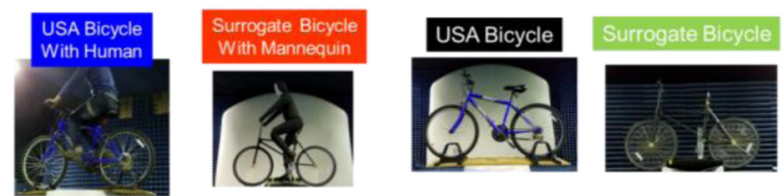

(a)

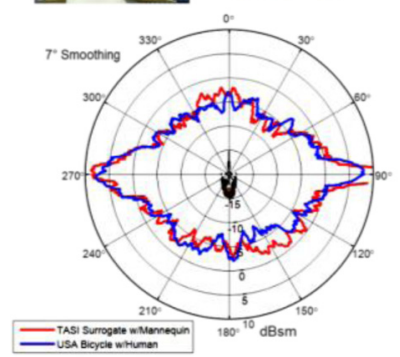

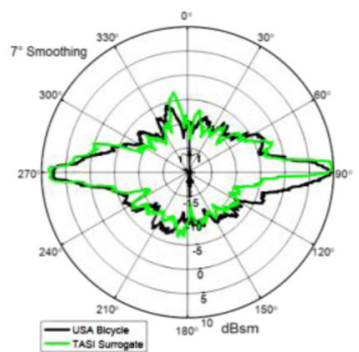

(b)
Notes: (a) RCS pattern comparison between real human riding a real bicycle and surrogate bicyclist attaching on the surrogate bicycle; (b) RCS pattern comparison between real bicycle and surrogate bicycle
Figure 20 Micro-Doppler response of pedaling legs with stationary wheels of the surrogate bicycle
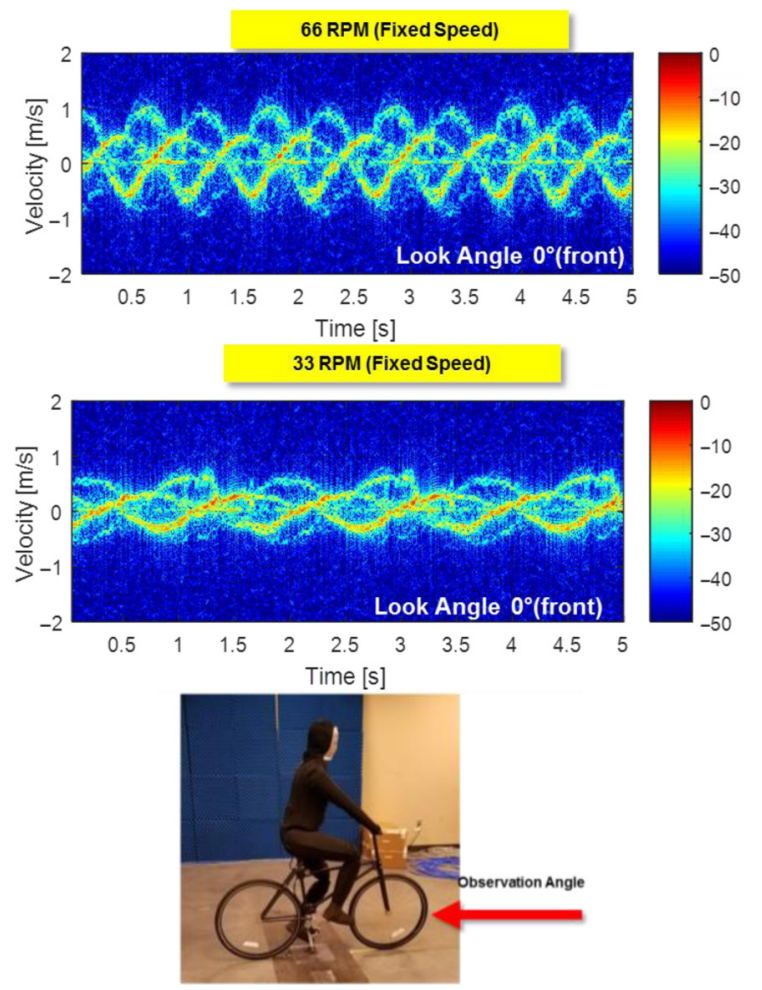

Figure 21 Micro-Doppler measurement of rotating surrogate wheel from behind
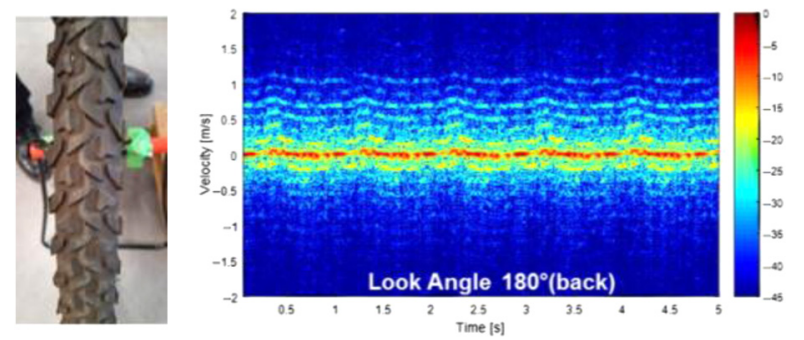

\section{Verification and automatic emergency braking systems system performance testing}

Verification and bicyclist AEB performance testing were conducted in the summer of 2015 and 2016. A production vehicle equipped with a bicyclist $A E B$ system was used for the verification and bicyclist AEB performance testing.

A complete set of bicyclist crash scenarios has been presented in Section 3 and Section 4. Among those, however, not every scenario is testable in the field because some scenarios are beyond the capability of the existing bicyclist AEB systems based on the vehicle user manual. The setup of the testing system is shown in Figure 22. It consists of: 
Figure 22 Overall test setup

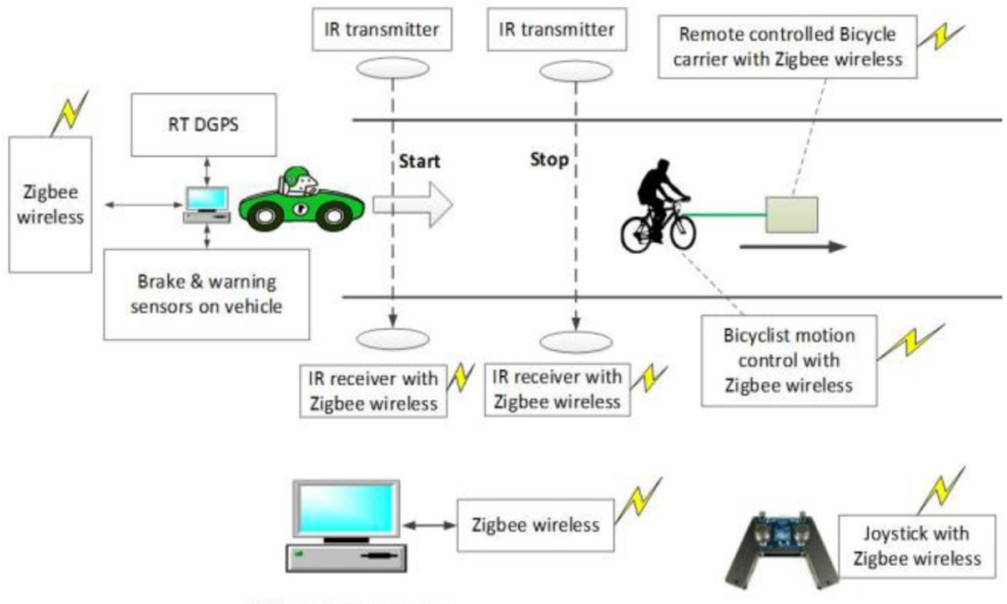

Main control computer

- differential GPS and data recording equipment installed on the test vehicle;

- a remotely controlled surrogate bicyclist;

- a remotely controlled bicycle transport platform;

- two infrared sensors called Start sensor and Stop sensor (the Start sensor is used to trigger the start motion of the surrogate bicyclist and the bicycle carrier, and the Stop sensor is only used for the scenario of crossing the road), to trigger the stop of surrogate bicyclist motion;

- a joystick controller for controlling the platform motion direction; and

- a central control computer.

All communication between the components is conducted wirelessly through a Zigbee network.

The differential GPS is used to measure the motion profile and the motion speed of the testing vehicle. The data recording equipment is designed to capture all AEB-related signals from the vehicle, which include brake pedal motion signal, brake light signal and AEB audio warning. The surrogate bicyclist is mounted on a sled. The bicycle carrier is designed to pull the sled moving along or crossing the road.

About 300 test runs were conducted and data were recorded. Table XII shows the actual testing scenarios obtained from the vehicle user manual. The range of vehicle speed is from $10 \mathrm{mph}$ to $60 \mathrm{mph}$ in an increment of $5 \mathrm{mph}$. The bicycle moving speed and mannequin leg pedaling speed are set at different speeds based on the testing scenarios, i.e. $4.1 \mathrm{~m} / \mathrm{s}$ ( 25 percentile),
$5.6 \mathrm{~m} / \mathrm{s}$ (average speed) and $6.9 \mathrm{~m} / \mathrm{s}$ (75 percentile) for moving along the road scenarios; $5.2 \mathrm{~m} / \mathrm{s}$ (average) for crossing the road with ride through scenarios; and $3.5 \mathrm{~m} / \mathrm{s}$ for crossing the road from ride out scenarios. For each vehicle test run, the DGPS data, sensor data and video data are captured. All test data have been processed and used for the AEB system scoring and benefit analysis. Two field testing photos for along the road scenario and crossing the road scenario are shown in Figures 23 and 24, respectively.

The purpose of the testing is not to evaluate the performance of the AEB system, but to check how to conduct the testing according to the desired test scenarios with the developed surrogate bicyclist and the associated hardware setup and software tools.

\section{Conclusion}

The development of testing scenarios and surrogate bicyclist system for the evaluation of bicyclist AEB systems has been described in this paper. Based on the analysis of the harmonized GES/FARS crash data, a set of five crash scenarios has been recommended for the performance testing of bicyclist AEB systems. Combined with TASI 110-car NDD, crash environmental factors including lighting conditions, obscuring objects, vehicle speed and bicyclist speed have been determined. The surrogate bicyclist was designed to represent the visual and radar characteristics of the real bicyclists in the USA. The height of the bicycle rider mannequin is $173 \mathrm{~cm}$, representing the

Table XII Scenarios for field testing

\begin{tabular}{lllll}
\hline Vehicle motion & Bicyclist motion & Bicyclist speed & Light condition & Vehicle speed \\
\hline Straight & Away from the Vehicle & $5.6 \mathrm{~m} / \mathrm{s}$ (average) & Daylight & 10 to $60 \mathrm{mph}$ with $5 \mathrm{mph}$ increment \\
& & $6.9 \mathrm{~m} / \mathrm{s}(75 \%)$ & \\
& Crossing & $4.1 \mathrm{~m} / \mathrm{s}(25 \%)$ & \\
& & $5.2 \mathrm{~m} / \mathrm{s}$ (Ride through) & \\
& Stationary & $3.5 \mathrm{~m} / \mathrm{s}$ (Ride out) & \\
\hline
\end{tabular}


Figure 23 Along the road testing scenario

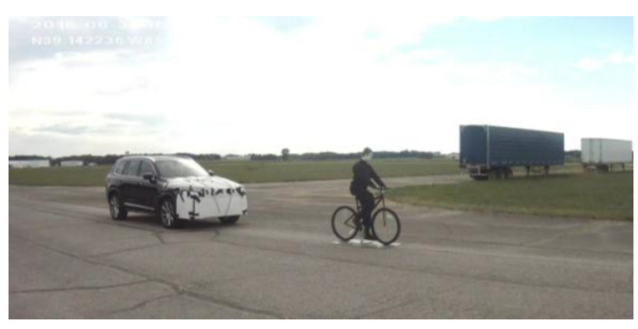

Note: Vehicle speed: $60 \mathrm{mph}$ and bicycle speed: $5.6 \mathrm{~m} / \mathrm{s}$

Figure 24 Crossing the road with constant speed testing scenario

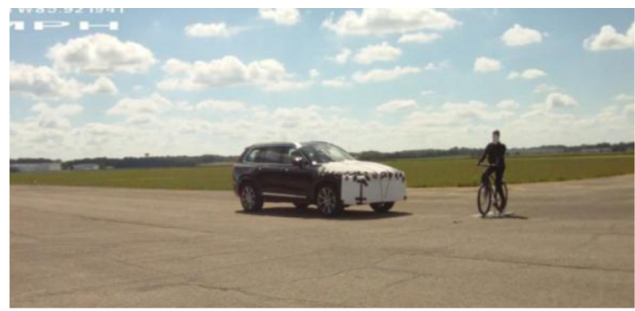

Note: Vehicle speed: $30 \mathrm{mph}$ and bicycle speed: $5.2 \mathrm{~m} / \mathrm{s}$

weighted height of 50th percentile US male and female adults. The size and shape of the surrogate bicycle were determined as 26-inch (660-mm) diameter wheel and mountain/road bicycle frame, respectively. Both leg pedaling motion and wheel rotation are suggested to produce the proper microDoppler features and support the camera-based AEB systems. Based on the analysis of the clothing color from 1,905 bicyclists in the TASI 110-car naturalistic driving study, black color has been determined as the representative color for both upper clothing (RGB: 57,60,67) and lower clothing (RGB: 44,42,46). The developed surrogate bicyclist also has the same $77 \mathrm{GHz}$ RCS as the real bicycle and bicycle rider from a 360-degree view. The developed scenarios, bicyclist surrogate and testing hardware and software tools have been verified in testing on a test track. The vehicle testing results have been shared with Society of Automotive engineers (SAE) Active Safety Standard Committee for the development of the SAE recommended practice for the active safety bicycle test surrogate targets.

\section{References}

Belgiovane, D. and Chen, C.C. (2017), "Micro-Doppler characteristics of pedestrians and bicycles for automotive radar sensors at $77 \mathrm{GHz}$ ", 2017 11th European Conference on Antennas and Propagation (EUCAP), IEEE, Paris, pp. 2912-2916.
CDC (2018), "Anthropometric reference data for children and adults: United States, 2011-2014", available at: www.cdc. gov/nchs/data/series/sr_03/sr03_039.pdf

CDN (2018), "(Euro NCAP) TEST PROTOCOL - AEB VRU systems", available at: https://cdn.euroncap.com/ media/26997/euro-ncap-aeb-vru-test-protocol-v20.pdf

den Camp, O.O., van Montfort, S., Uittenbogaard, J. and Welten, J. (2017), "Cyclist target and test setup for evaluation of cyclist-autonomous emergency braking", International fournal of Automotive Technology, Vol. 18 No. 6, pp. 1085-1097.

Fu, L., Tian, R., Li, L., Chen, Y. and Sherony, R. (2017), "Bicycle speed analysis for assessment of bicyclist precollision system", 25th International Technical Conference on the Enhanced Safety of Vehicles (ESV) National Highway Traffic Safety Administration, Washington, DC.

MacAlister, A. and Zuby, D.S. (2015), Cyclist Crash Scenarios and Factors Relevant to the Design of Cyclist Detection Systems, Insurance Institute for Highway Safety, Arlington, VA.

Milne, A. and Melin, M. (2014), "Bicycling and walking in the United States: 2014 benchmarking report”.

NHTSA (2016), "Fatal motor vehicle crashes: overview", available at: www.nhtsa.gov/press-releases/usdot-releases2016-fatal-traffic-crash-data

NHTSA (2018), "Traffic safety facts, bicyclists and other cyclists", available at: https://crashstats.nhtsa.dot.gov/Api/ Public/Publication/812382

Reynolds, C.C., Harris, M.A., Teschke, K., Cripton, P.A. and Winters, M. (2009), "The impact of transportation infrastructure on bicycling injuries and crashes: a review of the literature", Environmental Health, Vol. 8 No. 1, p. 47.

Rosen, E. (2013), "Autonomous emergency braking for vulnerable road users", Proceedings of IRCOBI Conference, pp. 618-627.

Sherony, R., Tian, R., Chien, S., Fu, L., Chen, Y. and Takahashi, H. (2016), "Pedestrian/bicyclist limb motion analysis from 110-car TASI video data for autonomous emergency braking testing surrogate development", $S A E$ International Fournal of Transportation Safety, Vol. 4 No. 1, pp. 113-120.

Statista (2018), "Number of cyclists/bike riders within the last 12 months in the United States from spring 2008 to spring 2017 (in millions)", available at: www.statista. com/statistics/227415/number-of-cyclists-and-bike-ridersusa/

Takahashi, K., Kuriya, Y. and Morie, T. (2010), "Bicycle detection using pedaling movement by spatiotemporal Gabor filtering”, TENCON 2010-2010 IEEE Region 10 Conference, IEEE, pp. 918-922.

TNO (2018), "CATS deliverable 3.4: CATS/4a bicyclist target specifications", available at: https://repository.tudelft.nl/ view/tno/uuid:f2ea4a8b-022a-402f-8251-0faff9c4a591/

Wojek, C., Walk, S. and Schiele, B. (2009), "Multi-cue onboard pedestrian detection", 2009. CVPR 2009. IEEE Conference on Computer Vision and Pattern Recognition, IEEE, Miami, FL, pp. 794-801. 
Yi, Q., Chien, S., Fu, L., Li, L., Chen, Y., Sherony, R. and Takahashi, H. (2017), "Clothing color of surrogate bicyclist for pre-collision system evaluation", 2017 IEEE Intelligent Vehicles Symposium (IV), IEEE, Los Angeles, CA, pp. 304-309.

Yi, Q., Chien, S., Brink, J., Niu, W., Li, L., Chen, Y., Chen, C. C., Sherony, R. and Takahashi, H., (2016), "Development of bicycle surrogate for bicyclist pre-collision system evaluation".
Yi, Q., Chien, S., Brink, J., Chen, Y., Li, L., Good, D., Chen, C.C. and Sherony, R. (2014), "Mannequin development for pedestrian pre-Collision System evaluation", 2014 IEEE 17th International Conference on Intelligent Transportation Systems (ITSC), IEEE, pp. 1626-1631.

\section{Corresponding author}

Lingxi Li can be contacted at: LL7@iupui.edu

For instructions on how to order reprints of this article, please visit our website:

www.emeraldgrouppublishing.com/licensing/reprints.htm

Or contact us for further details: permissions@emeraldinsight.com 